\title{
Re-defining Technical Reports
}

\author{
A refocused scope will infuse new technology into the regular research section of Nature Medicine and strengthen \\ the synergy between regular research articles and Technical Reports.
}

W e have recently witnessed an explosion of new technologies that may help us grapple with one of the biggest challenges blocking comprehensive understanding of human afflictions including neurodegenerative diseases, autoimmune diseases, and cancer: their heterogeneity in terms of etiology, progression and response to therapy. Examples of these technologies include genomeediting methods; single-cell technologies; approaches for comprehensively mapping and deciphering alterations in the genome, epigenome, proteome and metabolome; and protocols for generating primary human cells of particular lineages from other somatic cell types. Many of these techniques have undergone rapid optimization, and their cost and complexity in terms of implementation has plummeted while their availability has skyrocketed. As a result, we are at a stage at which researchers are able to apply these sophisticated approaches to reveal substantive insight into the biological basis of disease susceptibility, response or resistance to therapy, and to identify new drug targets. The aggregate potential of these new technologies to further translational research is astounding.

Thus, during an end-of-year assessment of the papers published in Nature Medicine and other journals, we took stock of how we can best welcome and feature papers using these approaches in the regular research and Technical Reports sections of the journal. One thing that became clear is that papers that apply cutting-edge-but previously described-technologies to discover and explore substantive new biological insights into disease should be featured in the regular research section of the journal rather than in the Technical Reports section. The other is that this was an opportunity to revisit our vision for the Technical Reports section of Nature Medicine.

First referred to by this name in Nature Medicine in May 2003 (and as 'New Technology' before that), Technical Reports have covered an incredible spectrum of preclinical and clinical topics and applications. In the last year alone this section included papers describing a noninvasive way of measuring intraocular pressure, an extracorporeal blood-cleansing device, a peptide for depleting myeloid-derived suppressor cells from mice, a drug delivery approach designed to amplify the effects of chemotherapy and radiation, and a method for generating and interpreting whole-exome sequencing data from formalinfixed paraffin-embedded human tumor tissues, among others. But given the large increase in the size of this section-we published 13 Technical Reports in 2004 and 29 a decade later in 2014-together with our desire to forge tighter conceptual synergy between Technical Reports and regular research articles in the journal, we have sharpened our criteria for their publication. These new criteria will soon to be added to our Guide to Authors: "A Technical Report presents primary research data on a new technique or method that uniquely enables new preclinical or clinical research of very broad interest. It may present a new biological discovery to demonstrate the utility of the technique, but substantive biological conceptual insight is not a requirement."

One key point is that a Technical Report will focus on describing, validating and benchmarking a fundamentally new technique, method or approach that is of very wide interest among our broad biomedical readership. Its main thrust will be the novel method and demonstrating its superiority to existing methods. Extensive new biological conceptual insight will remain a key criterion for regular research articles, but not for Technical Reports. Of course, biological insight generated by experiments needed to validate or demonstrate the superiority of a method may be included in a Technical Report. For example, a paper describing an imaging method that is twofold more sensitive than any existing method may need to use it to show that it can reveal cellular processes previously invisible to other methods. But a Technical Report need not present an exhaustive follow-up investigation of the molecular mechanisms underpinning these newly revealed processes.

The second key aspect is a focus on techniques that enable preclinical or clinical research. This will bring the Technical Reports section into closer conceptual synergy with the regular research section of the journal. The ideal Nature Medicine Technical Report will present a method; subsequent papers applying this method to discover new insights into human disease might be considered for publication in the regular research section of the journal. This re-focusing of the section on research-enabling methods means that some papers previously considered for publication in this section-those describing methods for surgery, diagnosis, and drug delivery come to mind-will no longer fit the Technical Report criteria. Some of these, particularly those using new technologies to achieve compelling therapeutic or diagnostic advances of broad interest, may be considered for the main research section of the journal. But for others, the editors of Nature Medicine will, when editorially applicable and when given permission by the authors, consult colleagues at related Nature Research Journals with the goal of providing authors informed guidance on the possibility of transferring the manuscript.

This effort to forge greater synergy between the Technical Reports and regular research sections also means that as of January 1, 2015 Technical Reports will be handled by the six in-house editors, each of whom currently handle regular research papers in their areas of disease expertise. As such, we bid farewell to Diane Gershon, who for many years has done a fantastic job handling Technical Reports for Nature Medicine. We thank Diane for all of her efforts, and we wish her the best of luck in her future endeavors.

As with all new policies and procedures, please do not hesitate to send questions to medicine@us.nature.com. 